\title{
Etude Bibliographique et Phytochimique de Quelques Plantes Médicinales Utilisées Pour Le Traitement de Certaines Maladies par les Tradipraticiens de la Zone de l'Azawagh au Niger
}

\author{
Moctar Chaibou, PhD \\ Abdoul Nasser Moussa Bamba, PhD \\ Idrissa Moussa, MC \\ Amadou Tidjani Ilagouma, MC \\ Ikhiri Khalid, Pr
}

Université Abdou Moumouni, Faculté des Sciences et Techniques,

Département de Chimie, Laboratoire des Substances Naturelles et Synthèse Organique (LASNASO), Niamey, Niger

URL:http://dx.doi.org/10.19044/esj.2020.v16n6p126

\section{Résumé}

Une enquête ethnobotanique menée dans la zone de l'Azawagh au Niger a permis de recenser et sélectionner trente (30) plantes contre diverses pathologies. Le but de ce travail est de justifier l'utilisation traditionnelle de ces plantes par les tradipraticiens sur la base d'une approche bibliographique. Un criblage phytochimique ainsi qu'une documentation approfondie (rapport d'enquête, articles, thèses...) ont servi de méthodologie. L'analyse phytochimique a permis de mettre en évidence plusieurs familles de métabolites secondaires ayant un lien avec les usages thérapeutiques des plantes utilisées. De même, la revue bibliographique a montré des usages similaires de ces plantes dans plusieurs pays. La présence des métabolites secondaires et les usages similaires justifient largement l'utilisation de ces plantes médicinales dans la zone de l'Azawagh.

Mots clés : Justification, Utilisations, Plantes Médicinales, Azawagh, Niger 


\title{
Bibliogrphic and Phytochemical Study of Some Medicinal Plants in the Treatment of Certain Diseases by Traditional Healers in the Azawagh Zone in Niger
}

\author{
Moctar Chaibou, PhD \\ Abdoul Nasser Moussa Bamba, PhD \\ Idrissa Moussa, MC \\ Amadou Tidjani Ilagouma, MC \\ Ikhiri Khalid, Pr
}

Université Abdou Moumouni, Faculté des Sciences et Techniques, Département de Chimie, Laboratoire des Substances Naturelles et Synthèse Organique (LASNASO), Niamey, Niger

\begin{abstract}
An ethnobotanical survey carried out in the zone of the Azawagh (Niger) permitted to count and to select thirty (30) plants against various pathologies. The aim of this work is to justify the use of these plants by traditional healers on the basis of a bibliographic approach. A phytochemical screening as well as an extensive documentation (survey report, articles, thesis ...) served as methodology. The phytochemical screening revealed the presence of several secondary metabolites families having a tie with the therapeutic uses of the plants used. In the same way, the bibliographic study showed similar uses of these plants in several countries. The presence of the secondary metabolites and the similar uses justify the use of these medicinal plants in the region of the Azawagh.
\end{abstract}

Keywords: Justification, Uses, Medicinals Plants, Azawagh, Niger

\section{Introduction}

De tous temps, l'Homme a entretenu des relations étroites avec les plantes qui l'entouraient. Ces plantes ont été utilisées à des fins diverses. Au début l'Homme s'en servait pour se nourrir. Au fil des temps ses connaissances s'élargissant, il leurs trouva d'autres utilités telles que matériaux aux usages multiples (poison de pêche ou chasse, lit, stimulant...) et comme médicaments, afin de soigner diverses maladies (Lee, 2004 ; Sofowara, 2010). 
Les expériences se transmettant de génération en génération, le pouvoir thérapeutique des plantes s'améliore dans de nombreux pays (Giani, 2006). Dans les pays en développement en général et ceux d'Afrique en particulier l'emploi des plantes médicinales persiste suite à la rareté et l'inaccessibilité des médicaments modernes par la majorité de la population (Dibong et al., 2011 ; Cisse et al., 2016). L'usage médicinal de ces plantes se faisait de manière empirique (Nacoulma, 1996). Cette utilisation est basée sur des croyances et des pratiques socio-culturelles. En effet, on ignorait tout de la composition chimique et le rôle des composés chimiques dans l'activité thérapeutique des différentes espèces médicinales utilisés tous les jours par les populations, pour les soins de santé (N'guessan et al., 2009).

Au Niger, le taux de couverture sanitaire est faible (FAD, 2001) surtout en milieu rural ce qui favorise une large présence de la pharmacopée traditionnelle. L'intérêt suscité par cette pharmacopée a conduit à une enquête ethnobotanique dans la zone de l'Azawagh par une équipe pluridisciplinaire composée de spécialistes en pharmacopée, de médecin, de biologiste et de chimiste. Cette enquête qui cadre avec le souci du gouvernement nigérien de rechercher un nouveau système de prestations sanitaires adapté à nos réalités a permis de répertorier et identifier plusieurs espèces végétales ainsi que leurs usages thérapeutiques (Ikhiri et al.,2000). Trente (30) d'entre elles ont été sélectionnées sur la base de la fréquence de leurs utilisations afin de les soumettre à une étude bibliographique pour savoir si oui ou non leurs utilisations par les tradipraticiens peuvent être justifiées. Pour atteindre l'objectif visé à savoir la justification scientifique des usages des plantes par la population de l'Azawagh, des études à caractères bibliographiques sont menées. Ces différentes plantes présentes également dans d'autres pays, leurs utilisations répétitives et régulières sont un indice important montrant leurs efficacités et justifiant leurs usages. À cela, il faut ajouter des études scientifiques déjà réalisées par d'autres auteurs indiquant certaines activités biologiques. Un criblage phytochimique a également été effectué. Les métabolites secondaires entrant dans la composition de ces plantes sont mis en évidence. Ces données phytochimiques permettent une meilleure connaissance des familles des molécules bioactives de ces plantes et pourraient contribuer éventuellement à la justification de leurs activités thérapeutiques.

\section{Matériel et méthodes}

\subsection{Matériel}

\subsubsection{Zone d'étude}

Avec une superficie de $420000 \mathrm{~km} 2$, l'Azawagh est une vaste zone située au Nord-Ouest du Niger, entre le désert du Sahara et la vallée fluviale du Niger (Figure 1). C'est une vaste zone de pâturages, au Nord de la limite 
entre la zone de cultures et la zone pastorale, à l'Ouest du massif de l'Aïr qui s'étend jusqu'à la frontière avec le Mali (Bernus, 1970).

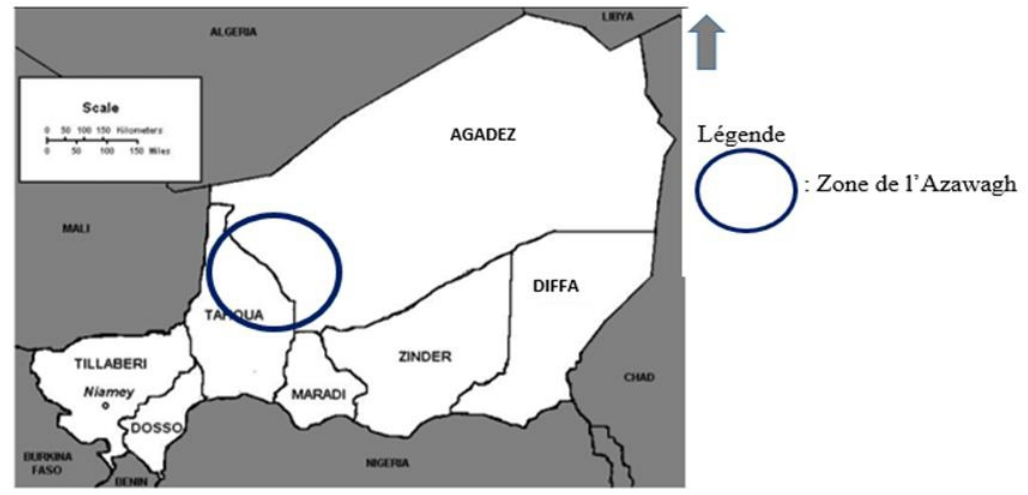

Figure 1 : Carte de la région de l'Azawagh (Bernus, 1970).

\subsubsection{Espèces végétales}

Le matériel végétal est constitué de trente (30) plantes. Ces espèces végétales ont été récoltées pendant les mois d'Août et Septembre 2018 puis séchées à l'ombre à la température ambiante. L'identification a été effectuée au département de biologie. Les différents parties (feuilles, tiges, fleurs, écorces, racines) de ces plantes ont été lavées, séchées et réduites en poudre.

\subsection{Méthodes}

Deux (2) types d'activités sont menés pendant les travaux : la recherche documentaire et le criblage phytochimique.

\subsubsection{Enquête ethnobotanique}

Apres avoir informé les autorités coutumières de l'objet de notre mission, une liste de tradipraticiens nous a été dressée (52 guérisseurs), puis un guide qui nous met en contact avec ces derniers. Les entretiens ont lieu tout moment de la journée selon la disponibilité de l'interlocuteur et notre heure d'arrivée dans la localité. L'entretien avec les guérisseurs a porté sur les maladies les plus fréquemment traitées, les plantes utilisées pour le traitement ainsi que le mode d'emploi.

\subsubsection{Criblage phytochimique}

Le criblage phytochimique s'est déroulé dans le Laboratoire de recherche du Département de Chimie de la Faculté des Sciences et Techniques de l'Université Abdou Moumouni de Niamey suivant un protocole standard de criblage chimique établi par Békro et al.,(2007) et Bruneton (1999). Les extraits aqueux ont été obtenus par macération dans de l'eau distillée pendant 48 heures. Après filtration sur coton hydrophile, les filtrats sont évaporés et 
concentrés à l'aide d'un bain de sable. Les composés recherchés étaient les alcaloïdes, les tanins, les saponosides, les flavonoïdes, les quinones, les stéroïdes et les terpènes.

\subsubsection{Recherche documentaire}

La recherche documentaire est effectuée à travers l'internet et dans les bibliothèques de la Faculté des Sciences et Techniques, la Faculté d'Agronomie, l'IRSH, l'AGRYMET et du Ministère de l'Agriculture et l'Elevage à partir d'une documentation approfondie et les données disponibles (rapport d'enquêtes, articles, thèses...).

\section{Résultats et discussion}

\subsection{Enquête ethnobotanique}

L'enquête ethnobotanique a permis d'inventorier 86 plantes. Par la suite, 30 plantes ont été sélectionnées sur la base de leur forte utilisation par la population de l'Azawagh. Les noms scientifiques et locaux des trente (30) plantes sélectionnées (Tableau 1) sont établis grâce à l'ouvrage de Frabegues (1972).

Tableau 1 : Noms des espèces végétales sélectionnées

\begin{tabular}{|c|c|c|c|}
\hline Familles & Noms scientifiques & $\begin{array}{c}\text { Noms } \\
\text { Tamasheq }\end{array}$ & $\begin{array}{c}\text { Noms } \\
\text { Haoussa }\end{array}$ \\
\hline Amaranthaceae & Achyrantes aspera Linn. & Mazergaz & $\begin{array}{c}\text { Machi } \\
\text { kadangare }\end{array}$ \\
\hline Annonaceae & Zizyphus mauritiana Lam. & Ajen & Magaria \\
\hline \multirow[b]{2}{*}{ Asclepiadaceae } & Calotropis procera (Aiton) W.T.Aiton & Tirza & Tumfafiya \\
\hline & Tamarindus indica L. & Tchmia & Tsamiya \\
\hline Balanitaceae & Balanites aegyptiaca (L.) Delile & Aboragh & Aduwa \\
\hline Burseraceae & Commiphora africana Engl Rich A. & Adaraz & Iskitshi \\
\hline \multirow{3}{*}{ Caesalpinaceae } & Bauhinia rufrescens Lam. & Taedeyni & Jiga \\
\hline & Cassia italica (Mill.)Spreng. & Agargar & Hilakso \\
\hline & Tribulus terrestris L. & Ageroff & Tsaydo \\
\hline \multirow{4}{*}{ Capparidaceae } & Boscia senegalensis (Pers.) ex Poir. & Taedaent & Anza \\
\hline & Cadaba glandulosa Forssk. & Teist & Taïs \\
\hline & Cadaba farinosa Forssk. & Abago & Bagayi \\
\hline & Maerua crassifolia Forssk. & Agar & dirga \\
\hline \multirow{4}{*}{ Combretaceae } & Combretum glutinosum Perr. ex DC. & Akalafa & Taramniya \\
\hline & Combretum micranthum G.Don & Tukuboult & Geza \\
\hline & Guiera senegalensis J.F. Gmel & Asubara & Sabara \\
\hline & Combretum glutinosum Perr. ex DC. & Akalafa & Taramniya \\
\hline Cucurbitaceae & Citrillus colocynthis (L.) Schrad. & Taegaellet & Guna \\
\hline \multirow[b]{2}{*}{ Euphorbiaceae } & Chrozophora brocchiana (Vis.) Schweinf & Aflinjid & Damagi \\
\hline & Euphorbia hirta L. & Talagh & Nono kurtchia \\
\hline Labiaceae & Sclerocarya birrea (A.Rich.) Hochst. & Tiwila & Danya \\
\hline Liliaceae & Allium sativum L. & Tafarnua & Tafarnua \\
\hline Lythraceae & Lawsonia inermis L. & Enila & Lalle \\
\hline Malvaceae & Waltheria indica $\mathrm{L}$. & Amatrak & Yankufa \\
\hline
\end{tabular}




\begin{tabular}{|c|l|l|l|}
\hline \multirow{2}{*}{ Mimosaceae } & $\begin{array}{l}\text { Acacia albida Willd. ex Delile subsp. } \\
\text { adstringens }\end{array}$ & Atis & Gawo \\
\cline { 2 - 4 } & Acacia nilotica (L.) Willd. ex Delile & Tiggaert & Bagaruwa \\
\hline Myrthaceae & Eugenia caryophyllata Thunb. & Inagalfiman & Karanfani \\
\hline Poaceae & Cymbopogon schoenantus Spreng. & Tebaremt & Tsabre \\
\hline Solanaceae & Ocinum canun Sims & Silmi & Kimba \\
\hline Sterculiaceae & Xylopia aethiopica (Dunal) A.Rich & Elian & Kimba \\
\hline Zingiberaceae & Zingiber officinale Roscoe & Kuturu yagi & Tchitta \\
\hline
\end{tabular}

\subsection{Résultats du criblage phytochimique}

Les résultats du criblage phytochimique sont inscrits dans le tableau 2. On note que toutes les plantes contiennent des tanins en dehors de T. terrestris, B. senegalensis et Z. officinale. Seules A. nilotica, C. italica et C. farinosa ne contiennent pas de flavonoïdes. Toutes les plantes à l'exception de A. nilotica, B. rufrescens, C. colocynthis et Z. officinale ne renferment pas de saponosides. S. birrea et L. inermis sont les plus riches en quinones. C. farinosa et $\mathrm{G}$. senegalensis ne contiennent pas de stéroïdes et terpènes. On note également l'absence des alcaloïdes dans A. nilotica, A. sativum, B. rufrescens, C. farinosa, C. africana, E. caryophyllata et Z. officinale.

Tableau 2 : Résultats du criblage chimique des trente (30) plantes.

\begin{tabular}{|c|c|c|c|c|c|c|c|c|}
\hline \multirow{2}{*}{$\begin{array}{l}\text { Espèces } \\
\text { végétales }\end{array}$} & \multirow[t]{2}{*}{ Organes } & \multicolumn{2}{|c|}{ Alcaloïde } & \multirow[t]{2}{*}{ Tanin } & \multirow[t]{2}{*}{ Flavonoïde } & \multirow[t]{2}{*}{ Saponoside } & \multirow[t]{2}{*}{ Quinone } & \multirow{2}{*}{$\begin{array}{l}\text { Stéroïde- } \\
\text { terpène }\end{array}$} \\
\hline & & M. & D. & & & & & \\
\hline $\begin{array}{l}\text { Acacia } \\
\text { albida }\end{array}$ & $\begin{array}{l}\text { Feuille } \\
\text { Tige } \\
\text { Fruit }\end{array}$ & $\begin{array}{l}++ \\
- \\
+\end{array}$ & $\begin{array}{l}++ \\
+ \\
+\end{array}$ & $\begin{array}{l}++ \\
++ \\
++\end{array}$ & $\begin{array}{l}+ \\
++ \\
+\end{array}$ & $\begin{array}{l}+ \\
+ \\
+\end{array}$ & $\begin{array}{l}- \\
- \\
-\end{array}$ & $\begin{array}{l}+ \\
+ \\
+\end{array}$ \\
\hline $\begin{array}{l}\text { Acacia } \\
\text { nilotica }\end{array}$ & $\begin{array}{l}\text { Feuille } \\
\text { Fruit }\end{array}$ & - & - & $\begin{array}{l}++ \\
+++\end{array}$ & - & - & - & + \\
\hline $\begin{array}{l}\text { Achyrantes } \\
\text { aspera }\end{array}$ & $\begin{array}{l}\text { Feuille } \\
\text { Tige } \\
\text { Racine } \\
\text { Fruit }\end{array}$ & $\begin{array}{l}- \\
- \\
- \\
+ \\
\end{array}$ & $\begin{array}{l}- \\
- \\
- \\
+\end{array}$ & $\begin{array}{l}- \\
- \\
+++ \\
- \\
\end{array}$ & $\begin{array}{l}- \\
- \\
+ \\
+\end{array}$ & $\begin{array}{l}- \\
+ \\
+ \\
+\end{array}$ & $\begin{array}{l}- \\
- \\
+ \\
+\end{array}$ & $\begin{array}{l}+ \\
+ \\
+ \\
+\end{array}$ \\
\hline $\begin{array}{l}\text { Allium } \\
\text { sativum }\end{array}$ & Bulbe & - & - & - & + & + & + & + \\
\hline $\begin{array}{l}\text { Balanites } \\
\text { aegyptiaca }\end{array}$ & $\begin{array}{l}\text { Feuille } \\
\text { Tige } \\
\text { Fleur } \\
\text { Ecorce } \\
\text { Pulpe } \\
\end{array}$ & $\begin{array}{l} \\
+ \\
+ \\
+ \\
++\end{array}$ & $\begin{array}{l}- \\
- \\
+ \\
++ \\
++\end{array}$ & $\begin{array}{l}+ \\
- \\
- \\
+ \\
-\end{array}$ & $\begin{array}{l}++ \\
+ \\
+ \\
+ \\
- \\
\end{array}$ & $\begin{array}{l}+ \\
+ \\
+ \\
+ \\
+ \\
\end{array}$ & $\begin{array}{l}- \\
- \\
+ \\
- \\
- \\
\end{array}$ & $\begin{array}{l}+ \\
+ \\
+ \\
+ \\
+\end{array}$ \\
\hline $\begin{array}{l}\text { Bauhinia } \\
\text { rufrescens }\end{array}$ & $\begin{array}{l}\text { Feuille } \\
\text { Tige } \\
\text { Fruit }\end{array}$ & $\begin{array}{l}- \\
- \\
-\end{array}$ & $\begin{array}{l}- \\
- \\
\end{array}$ & $\begin{array}{l}+ \\
++ \\
++\end{array}$ & $\begin{array}{l}+ \\
++ \\
++\end{array}$ & $\begin{array}{l}- \\
- \\
-\end{array}$ & $\begin{array}{l}- \\
+ \\
-\end{array}$ & $\begin{array}{l}+ \\
+ \\
+ \\
\end{array}$ \\
\hline $\begin{array}{l}\text { Boscia } \\
\text { senegalensis }\end{array}$ & $\begin{array}{l}\text { Feuille } \\
\text { Tige } \\
\text { Racine }\end{array}$ & $\begin{array}{l}- \\
+ \\
++\end{array}$ & $\begin{array}{l}- \\
++ \\
++\end{array}$ & $\begin{array}{l}- \\
- \\
-\end{array}$ & $\begin{array}{l}++ \\
+ \\
-\end{array}$ & $\begin{array}{l}+ \\
+ \\
+\end{array}$ & $\begin{array}{l}- \\
- \\
-\end{array}$ & $\begin{array}{l}+ \\
+ \\
+\end{array}$ \\
\hline
\end{tabular}

- : absence ; $:$ présence ; ++ : présence abondant ; +++ : présence très abondant ; M : Mayer ; D : Dragendoorf 
Tableau 2 : (Suite).

\begin{tabular}{|c|c|c|c|c|c|c|c|c|}
\hline \multirow{2}{*}{$\begin{array}{l}\text { Espèces } \\
\text { végétales }\end{array}$} & \multirow[t]{2}{*}{ Organes } & \multicolumn{2}{|c|}{ Alcaloïde } & \multirow[t]{2}{*}{ Tanin } & \multirow[t]{2}{*}{ Flavonoïde } & \multirow[t]{2}{*}{ Saponoside } & \multirow[t]{2}{*}{ Quinone } & \multirow{2}{*}{$\begin{array}{l}\text { Stéroïde- } \\
\text { terpène }\end{array}$} \\
\hline & & $\mathrm{M}$ & $\mathrm{D}$ & & & & & \\
\hline $\begin{array}{l}\text { Cadaba } \\
\text { glandulosa }\end{array}$ & $\begin{array}{l}\text { Feuille } \\
\text { Tige } \\
\text { Fruit }\end{array}$ & $\begin{array}{l}++ \\
- \\
+\end{array}$ & $\begin{array}{l}++ \\
- \\
++\end{array}$ & $\begin{array}{l}+ \\
- \\
-\end{array}$ & $\begin{array}{l}++ \\
- \\
++\end{array}$ & $\begin{array}{l}+ \\
+ \\
+\end{array}$ & $\begin{array}{l}- \\
- \\
-\end{array}$ & $\begin{array}{l}+ \\
+ \\
+\end{array}$ \\
\hline $\begin{array}{l}\text { Cadaba } \\
\text { farinosa } \\
\end{array}$ & Feuille & - & - & + & - & + & - & - \\
\hline $\begin{array}{l}\text { Calotropis } \\
\text { procera }\end{array}$ & $\begin{array}{l}\text { Feuille } \\
\text { Tige } \\
\text { Fleur } \\
\text { Fruit }\end{array}$ & $\begin{array}{l}- \\
- \\
- \\
+\end{array}$ & $\begin{array}{l}- \\
- \\
- \\
-\end{array}$ & $\begin{array}{l}+ \\
+ \\
+ \\
+\end{array}$ & $\begin{array}{l}+++ \\
- \\
+ \\
+++\end{array}$ & $\begin{array}{l}- \\
- \\
- \\
+\end{array}$ & $\begin{array}{l}- \\
- \\
- \\
-\end{array}$ & $\begin{array}{l}+ \\
+ \\
+ \\
+\end{array}$ \\
\hline Cassia italica & $\begin{array}{l}\text { Feuille } \\
\text { Tige } \\
\text { Fleur }\end{array}$ & $\begin{array}{l}- \\
- \\
-\end{array}$ & $\begin{array}{l}- \\
- \\
+\end{array}$ & $\begin{array}{l}+ \\
++ \\
++\end{array}$ & $\begin{array}{l}- \\
- \\
-\end{array}$ & $\begin{array}{l}- \\
- \\
+\end{array}$ & $\begin{array}{l}- \\
+ \\
-\end{array}$ & $\begin{array}{l}+ \\
+ \\
+\end{array}$ \\
\hline $\begin{array}{l}\text { Chrozophora } \\
\text { brocchiana }\end{array}$ & $\begin{array}{l}\text { Feuille } \\
\text { Tige } \\
\text { Fruit } \\
\end{array}$ & $\begin{array}{l}- \\
- \\
-\end{array}$ & $\begin{array}{l}+ \\
- \\
-\end{array}$ & $\begin{array}{l}+++ \\
+++ \\
+++\end{array}$ & $\begin{array}{l}++ \\
+ \\
+++\end{array}$ & $\begin{array}{l}- \\
+ \\
-\end{array}$ & $\begin{array}{l}- \\
- \\
-\end{array}$ & $\begin{array}{l}+ \\
+ \\
+\end{array}$ \\
\hline $\begin{array}{l}\text { Citrillus } \\
\text { colocynthis }\end{array}$ & Pulpe & + & + & + & + & - & - & ++ \\
\hline $\begin{array}{l}\text { Combretum } \\
\text { glutinosum }\end{array}$ & Feuille & + & - & ++ & ++ & + & - & + \\
\hline $\begin{array}{l}\text { Combretum } \\
\text { micranthum }\end{array}$ & $\begin{array}{l}\text { Feuille } \\
\text { Tige } \\
\text { Fruit }\end{array}$ & $\begin{array}{l}+ \\
+ \\
-\end{array}$ & $\begin{array}{l}- \\
+ \\
-\end{array}$ & $\begin{array}{l}+++ \\
+++ \\
+++\end{array}$ & $\begin{array}{l}+++ \\
++ \\
+++\end{array}$ & $\begin{array}{l}+ \\
+ \\
-\end{array}$ & $\begin{array}{l}- \\
- \\
++\end{array}$ & $\begin{array}{l}+ \\
+ \\
+\end{array}$ \\
\hline $\begin{array}{l}\text { Commiphora } \\
\text { africana }\end{array}$ & $\begin{array}{l}\text { Feuille } \\
\text { Tige } \\
\text { Racine } \\
\text { Ecorce }\end{array}$ & $\begin{array}{l}- \\
- \\
- \\
-\end{array}$ & $\begin{array}{l}- \\
- \\
- \\
-\end{array}$ & $\begin{array}{l}++ \\
+ \\
++ \\
++\end{array}$ & $\begin{array}{l}++ \\
+ \\
+ \\
++\end{array}$ & $\begin{array}{l}- \\
- \\
- \\
+\end{array}$ & $\begin{array}{l}- \\
- \\
+ \\
-\end{array}$ & $\begin{array}{l}+ \\
+ \\
+ \\
+\end{array}$ \\
\hline $\begin{array}{l}\text { Cymbopogon } \\
\text { schoenantus }\end{array}$ & $\begin{array}{l}\text { Racine } \\
\text { Partie } \\
\text { aérienne }\end{array}$ & $\begin{array}{l}+ \\
+\end{array}$ & $\begin{array}{l}+ \\
++\end{array}$ & $\begin{array}{l}++ \\
++\end{array}$ & $\begin{array}{l}- \\
+\end{array}$ & $\begin{array}{l}- \\
++\end{array}$ & $\begin{array}{l}- \\
-\end{array}$ & $\begin{array}{l}+ \\
+\end{array}$ \\
\hline $\begin{array}{l}\text { Eugenia } \\
\text { caryophyllata }\end{array}$ & Feuille & - & - & ++ & ++ & + & - & ++ \\
\hline $\begin{array}{l}\text { Euphorbia } \\
\text { hirta }\end{array}$ & $\begin{array}{l}\text { Plante } \\
\text { entière }\end{array}$ & - & + & ++ & + & + & + & + \\
\hline
\end{tabular}

- : absence $;+$ : présence ; ++ : présence abondant ; +++ : présence très abondant ; M : Mayer ; D : Dragendoorf 
Tableau 2 : (Suite et fin)

\begin{tabular}{|c|c|c|c|c|c|c|c|c|}
\hline \multirow{2}{*}{$\begin{array}{l}\text { Espèces } \\
\text { végétales }\end{array}$} & \multirow[t]{2}{*}{ Organes } & \multicolumn{2}{|c|}{ Alcaloïde } & \multirow[t]{2}{*}{ Tanin } & \multirow[t]{2}{*}{ Flavonoïde } & \multirow[t]{2}{*}{ Saponoside } & \multirow[t]{2}{*}{ Quinone } & \multirow{2}{*}{$\begin{array}{l}\text { Stéroïde- } \\
\text { terpène }\end{array}$} \\
\hline & & $\mathrm{M}$ & $\mathrm{D}$ & & & & & \\
\hline $\begin{array}{l}\text { Guiera } \\
\text { senegalensis }\end{array}$ & Feuille & + & + & +++ & + & + & - & - \\
\hline $\begin{array}{l}\text { Lawsonia } \\
\text { inermis }\end{array}$ & Feuille & + & + & + & ++ & + & ++ & + \\
\hline $\begin{array}{l}\text { Maerua } \\
\text { crassifolia }\end{array}$ & $\begin{array}{l}\text { Partie } \\
\text { aérienne }\end{array}$ & - & + & + & + & + & - & + \\
\hline $\begin{array}{l}\text { Ocinum } \\
\text { canun Sims }\end{array}$ & Feuille & + & + & + & + & + & - & + \\
\hline $\begin{array}{l}\text { Sclerocarya } \\
\text { birrea }\end{array}$ & $\begin{array}{l}\text { Feuille } \\
\text { Tige } \\
\text { Ecorce } \\
\text { Endocar } \\
\text { pe }\end{array}$ & $\begin{array}{l}+ \\
- \\
- \\
-\end{array}$ & $\begin{array}{l}- \\
- \\
- \\
-\end{array}$ & $\begin{array}{l}+++ \\
+++ \\
++ \\
-\end{array}$ & $\begin{array}{l}++ \\
++ \\
+++ \\
+\end{array}$ & $\begin{array}{l}+ \\
+ \\
+ \\
-\end{array}$ & $\begin{array}{l}++ \\
+ \\
++ \\
-\end{array}$ & $\begin{array}{l}+ \\
+ \\
+ \\
+\end{array}$ \\
\hline $\begin{array}{l}\text { Tamarindus } \\
\text { indica }\end{array}$ & $\begin{array}{l}\text { Feuille } \\
\text { Fruit } \\
\text { Ecorce }\end{array}$ & $\begin{array}{l}- \\
- \\
++\end{array}$ & $\begin{array}{l}- \\
- \\
+ \\
\end{array}$ & $\begin{array}{l}++ \\
- \\
+++ \\
\end{array}$ & $\begin{array}{l}++ \\
+ \\
++ \\
\end{array}$ & $\begin{array}{l} \\
+ \\
++\end{array}$ & $\begin{array}{l}- \\
- \\
-\end{array}$ & $\begin{array}{l} \\
++ \\
- \\
\end{array}$ \\
\hline $\begin{array}{l}\text { Tribulus } \\
\text { terrestris }\end{array}$ & Feuille & + & + & - & + & ++ & - & + \\
\hline $\begin{array}{l}\text { Waltheria } \\
\text { indica }\end{array}$ & $\begin{array}{l}\text { Plante } \\
\text { entière }\end{array}$ & + & - & ++ & + & ++ & + & + \\
\hline $\begin{array}{l}\text { Xylopia } \\
\text { aethiopica }\end{array}$ & $\begin{array}{l}\text { Feuille } \\
\text { Fruit } \\
\text { Ecorce }\end{array}$ & $\begin{array}{l}++ \\
+ \\
++\end{array}$ & $\begin{array}{l}+ \\
+ \\
+\end{array}$ & $\begin{array}{l}+ \\
- \\
-\end{array}$ & $\begin{array}{l}+ \\
+ \\
++\end{array}$ & $\begin{array}{l}+ \\
+ \\
++\end{array}$ & $\begin{array}{l}- \\
- \\
+\end{array}$ & $\begin{array}{l}+ \\
+ \\
-\end{array}$ \\
\hline $\begin{array}{l}\text { Zingiber } \\
\text { officinale }\end{array}$ & $\begin{array}{l}\text { Rhizom } \\
\mathrm{e}\end{array}$ & - & - & - & + & - & - & ++ \\
\hline $\begin{array}{l}\text { Zizyphus } \\
\text { mauritiana }\end{array}$ & $\begin{array}{l}\text { Feuille } \\
\text { Tige } \\
\text { Pulpe }\end{array}$ & $\begin{array}{l}- \\
- \\
-\end{array}$ & $\begin{array}{l}- \\
+ \\
+\end{array}$ & $\begin{array}{l}+++ \\
++ \\
++\end{array}$ & $\begin{array}{l}++ \\
++ \\
+++\end{array}$ & $\begin{array}{l}+ \\
- \\
-\end{array}$ & $\begin{array}{l}- \\
- \\
-\end{array}$ & $\begin{array}{l}+ \\
+ \\
+\end{array}$ \\
\hline
\end{tabular}

- : absence ; + : présence ; ++ : présence abondant ; +++ : présence très abondant ; $M$ : Mayer ; D : Dragendoorf

\subsection{Recherche documentaire}

La recherche documentaire a permis de faire un inventaire des données sur les usages des espèces végétales sélectionnées dans d'autres pays, leurs compositions chimiques et les activités biologiques liées à chaque composé chimique.

\subsubsection{Usages des plantes sélectionnées dans l'Azawagh et dans d'autres pays}

Les résultats concernant les usages des plantes dans l'Azawagh et dans d'autres pays sont consignés dans le tableau 3. Il ressort de ces résultats que les usages des plantes sélectionnées dans l'Azawagh sont similaires à ceux 
d'autres pays. Cependant on constate que d'autres usages sont spécifiques à la zone de l'Azawagh.

Tableau 3: Les usages médicinaux des trente (30) plantes dans l'Azawagh et les pays ayant des usages similaires.

\begin{tabular}{|c|c|c|c|c|}
\hline Espèces végétales & Organes utilisés & $\begin{array}{l}\text { Usages } \\
\text { l'Azawagh }\end{array}$ & $\begin{array}{lr}\text { Pays ayant des usages } \\
\text { similaires } \\
\text { l'Azawagh }\end{array}$ & Références \\
\hline \multirow{5}{*}{ Acacia albida } & \multirow[t]{3}{*}{ Feuilles } & Diarrhées & Benin, Burkina Faso & $\begin{array}{l}\text { Adjanohoun (1989), } \\
\text { Serene (2008 }\end{array}$ \\
\hline & & Maux de ventre & & \\
\hline & & Hémorroïdes & & \\
\hline & & Dysenterie & & \\
\hline & Tiges & Ictère & & \\
\hline \multirow{4}{*}{ Acacia nilotica } & \multirow{4}{*}{ Gousses } & Diarrhées & \multirow[b]{2}{*}{ Sénégal } & \multirow[b]{2}{*}{ Pousset (2004) } \\
\hline & & \begin{tabular}{|l|} 
Plaies, aphte \\
Billariogee \\
\end{tabular} & & \\
\hline & & \begin{tabular}{|l} 
B1lharziose \\
Dysenterie
\end{tabular} & Burkina Faso & Serene (2008) \\
\hline & & Stomatites & & \\
\hline Allium sativum & Bulbes & \begin{tabular}{|l|}
$\begin{array}{l}\text { Convulsions, vers } \\
\text { intestinaux }\end{array}$ \\
\end{tabular} & Benin & Adjanohoun (1989) \\
\hline \multirow{3}{*}{$\begin{array}{l}\text { Achyrantes } \\
\text { aspera }\end{array}$} & \multirow{3}{*}{ Plante entière } & Toux & Mauritanie & Hmeiada (2009) \\
\hline & & Fertilité & Inde & Hassan (2014) \\
\hline & & Syphilis & & \\
\hline \multirow{8}{*}{$\begin{array}{l}\text { Balanites } \\
\text { aegyptiaca }\end{array}$} & \multirow[b]{2}{*}{ Racines } & Rhume & Mauritanie & Hmeiada (2009) \\
\hline & & \begin{tabular}{|l|} 
morsures \\
venimeuses
\end{tabular} & \multirow{4}{*}{ Sénégal } & \multirow{4}{*}{ Pousset (2004) } \\
\hline & Fruits & vers intestinaux & & \\
\hline & \multirow{5}{*}{ Feuilles } & Coliques & & \\
\hline & & Ictère & & \\
\hline & & Hémorroïdes & & \\
\hline & & Edèmes & & \\
\hline & & Sinusite & & \\
\hline \multirow{6}{*}{$\begin{array}{l}\text { Bauhinia } \\
\text { rufrescens }\end{array}$} & Tiges & Lombalgies & & \\
\hline & \multirow{3}{*}{ Ecorces } & Hémorroïdes & & \\
\hline & & Diarrhées & $\begin{array}{l}\text { Burkina } \\
\text { Mauritanie }\end{array}$ & \begin{tabular}{|l} 
Hmeiada \\
Serene (2008)
\end{tabular} \\
\hline & & Maux de ventre & \multirow{3}{*}{ Burkina Faso } & \multirow{3}{*}{ Serene (2008) } \\
\hline & \multirow{2}{*}{ Feuilles } & Ictère & & \\
\hline & & Dysenterie & & \\
\hline
\end{tabular}


Tableau 3: (Suite).

\begin{tabular}{|c|c|c|c|c|}
\hline Espèces végétales & \begin{tabular}{|l} 
Organes \\
utilisés
\end{tabular} & \begin{tabular}{|ll} 
Usages & dans \\
l'Azawagh & \\
\end{tabular} & \begin{tabular}{|l|} 
Pays ayant des usages \\
similaires que l'Azawagh
\end{tabular} & Références \\
\hline \multirow{5}{*}{ Cassia italica } & Fleurs & Splénomégalie & & \\
\hline & \begin{tabular}{|l|} 
Racines, \\
fleurs \\
\end{tabular} & \begin{tabular}{|l|}
$\begin{array}{l}\text { ballonnement } \mathrm{de} \\
\text { ventre }\end{array}$ \\
\end{tabular} & & \\
\hline & \begin{tabular}{|l|} 
Feuilles \\
\end{tabular} & Maux de ventre & Sénégal & Pousset (2004) \\
\hline & \multirow[t]{2}{*}{ Racines } & Ictère & & \\
\hline & & Lèpre & & \\
\hline \multirow[t]{2}{*}{ Tribulus terrestris } & Feuilles & hémorragies & & \\
\hline & \begin{tabular}{|l|} 
Tiges \\
\end{tabular} & \begin{tabular}{|l|}
$\begin{array}{l}\text { impuissance } \\
\text { sexuelle }\end{array}$ \\
\end{tabular} & Egypte & Batanouny (1999) \\
\hline \multirow{4}{*}{ Boscia senegalensis } & Ecorces & hémorragies & & \\
\hline & \begin{tabular}{|l|} 
Feuilles \\
\end{tabular} & Maux de ventre & Sénégal & Pousset (2004) \\
\hline & \multirow[b]{2}{*}{ Racines } & Ictère & & \\
\hline & & Diarrhées & & \\
\hline \multirow{4}{*}{ Cadaba glandulosa } & \multirow{4}{*}{ Feuilles } & \begin{tabular}{|l|} 
Sinusite \\
\end{tabular} & & \\
\hline & & Bilharziose & & \\
\hline & & Asthme & Mali & Adjanohoun (1985) \\
\hline & & Diarrhées & & \\
\hline \multirow{4}{*}{ Cadaba farinosa } & & Maux de ventre & & \\
\hline & Feuilles & vers intestinaux & \multirow{2}{*}{ Inde } & \multirow[t]{2}{*}{ Umesh (2010) } \\
\hline & Racines & fatigue générale & & \\
\hline & Feuilles & Ictère & & \\
\hline \multirow{5}{*}{ Maerua crassifolia } & \multirow[b]{5}{*}{ Feuilles } & Toux & & \\
\hline & & Aphtes & & \\
\hline & & Ictère & Soudan & Kokwaro (1976) \\
\hline & & Paludisme & \multirow{2}{*}{ Nigeria } & \multirow[b]{2}{*}{ Sofowara (1986) } \\
\hline & & Diarrhées & & \\
\hline \multirow{4}{*}{ Calotropis procera } & \multirow{4}{*}{ Feuilles } & Sinusite & & \\
\hline & & \begin{tabular}{|l|} 
Brulures \\
\end{tabular} & & \\
\hline & & Toux & \multirow{2}{*}{ Benin } & \multirow{2}{*}{ Adjanohoun (1989) } \\
\hline & & Edèmes & & \\
\hline \multirow{4}{*}{$\begin{array}{l}\text { Chrozophora } \\
\text { brocchiana }\end{array}$} & \multirow{4}{*}{ Feuilles } & Maux de ventre & & \\
\hline & & CEdèmes & & \\
\hline & & Diarrhées & Benin & Adjanohoun (1989) \\
\hline & & Dysenterie & Mali & \begin{tabular}{|l} 
Adjanohoun \\
$(1985)$
\end{tabular} \\
\hline \multirow{4}{*}{ Euphorbia hirta } & Feuilles & Blennorragie & & \\
\hline & \multirow{3}{*}{ Plante entière } & Asthme & \multirow{3}{*}{ Nigeria } & \multirow{3}{*}{ Sofowara (1986) } \\
\hline & & Bronchite & & \\
\hline & & $\begin{array}{l}\text { maladies } \\
\text { respiratoires }\end{array}$ & & \\
\hline
\end{tabular}


Tableau 3: (Suite).

\begin{tabular}{|c|c|c|c|c|}
\hline Espèces végétales & $\begin{array}{l}\text { Organes } \\
\text { utilisés }\end{array}$ & $\begin{array}{l}\text { Usages dans } \\
\text { l'Azawagh }\end{array}$ & $\begin{array}{c}\text { Pays ayant des usages } \\
\text { similaires que } \\
\text { l'Azawagh }\end{array}$ & Références \\
\hline \multirow{2}{*}{$\begin{array}{l}\text { Citrillus } \\
\text { colocynthis }\end{array}$} & \multirow[t]{2}{*}{ Pulpe } & $\begin{array}{c}\text { morsures } \\
\text { venimeuses }\end{array}$ & & \\
\hline & & Dermatoses & Mauritanie & Hmeiada (2009) \\
\hline \multirow{5}{*}{$\begin{array}{l}\text { Combretum } \\
\text { glutinosum }\end{array}$} & Racines & Furoncle & & \\
\hline & Ecorces & Dysenterie & & \\
\hline & \multirow[t]{3}{*}{ Feuilles } & Maux de ventre & & \\
\hline & & Ictère & \multirow{2}{*}{ Sénégal } & \multirow{2}{*}{ Pousset (2004) } \\
\hline & & Paludisme & & \\
\hline \multirow{5}{*}{$\begin{array}{l}\text { Combretum } \\
\text { micranthum }\end{array}$} & \multirow{5}{*}{ Feuilles } & Dyspepsie & & \\
\hline & & Maux de ventre & & \\
\hline & & Ictère & Soudan & Kokwaro (1976) \\
\hline & & Fièvre & \multirow{2}{*}{ Sénégal } & \multirow{2}{*}{ Pousset (2004) } \\
\hline & & Plaie & & \\
\hline \multirow{4}{*}{$\begin{array}{c}\text { Guiera } \\
\text { senegalensis }\end{array}$} & \multirow[t]{2}{*}{ Racines } & Maux de ventre & & \\
\hline & & Ictère & & \\
\hline & \multirow[t]{2}{*}{ Feuilles } & Fièvre & \multirow{2}{*}{ Sénégal } & \multirow{2}{*}{ Pousset (2004) } \\
\hline & & Dysenterie & & \\
\hline \multirow{4}{*}{$\begin{array}{l}\text { Commiphora } \\
\text { africana }\end{array}$} & Feuilles & Toux & & \\
\hline & \multirow[t]{2}{*}{ Ecorces } & Dysenterie & & \\
\hline & & Maux de ventre & Nigeria & Sofowara (1986) \\
\hline & Tige & hémorragies & Djibouti & Abdallah (2013) \\
\hline \multirow{4}{*}{$\begin{array}{l}\text { Cymbopogon } \\
\text { schoenantus }\end{array}$} & \multirow[t]{4}{*}{ Feuilles } & Courbature & & \\
\hline & & Myalgie & & \\
\hline & & Ophtalmie & & \\
\hline & & Anuries & Soudan & Kokwaro (1976) \\
\hline \multirow{3}{*}{$\begin{array}{c}\text { Eugenia } \\
\text { caryophyllata }\end{array}$} & \multirow[t]{3}{*}{ Feuilles } & Rhume & & \\
\hline & & hémorragies & & \\
\hline & & Sinusite & Madagascar & Boiteau (1987). \\
\hline \multirow{2}{*}{ Lawsonia inermis } & \multirow[t]{2}{*}{ Feuilles } & Plaies & & \\
\hline & & Furoncle & Sénégal & Pousset (2004) \\
\hline \multirow{2}{*}{$\begin{array}{l}\text { Ocinum canun } \\
\text { Sims }\end{array}$} & Feuilles & Rhume & & \\
\hline & Plante entière & Toux & Madagascar & Boiteau (1987). \\
\hline \multirow{4}{*}{ Sclerocarya birrea } & Feuilles & Diarrhées & & \\
\hline & \multirow[t]{3}{*}{ Ecorces } & Ictère & & \\
\hline & & Paludisme & Mali & Adjanohoun (1985) \\
\hline & & Dysenterie & Sénégal & Kerharo (1974) \\
\hline \multirow{2}{*}{ Tamarindus indica } & \multirow[t]{2}{*}{ Fruit } & Paludisme & Sénégal & Pousset (2004) \\
\hline & & Fièvre & Benin & Adjanohoun (1989) \\
\hline
\end{tabular}


Tableau 3 : (Suite et fin).

\begin{tabular}{|c|c|c|c|c|}
\hline $\begin{array}{l}\text { Espèces } \\
\text { végétales }\end{array}$ & $\begin{array}{l}\text { Organes } \\
\text { utilisés }\end{array}$ & $\begin{array}{ll}\text { Usages } & \text { dans } \\
\text { l'Azawagh } & \\
\end{array}$ & $\begin{array}{l}\text { Pays ayant des usages } \\
\text { similaires que l'Azawagh }\end{array}$ & Références \\
\hline \multirow[t]{2}{*}{$\begin{array}{l}\text { Tamarindus } \\
\text { indica }\end{array}$} & \multirow[t]{2}{*}{ Fruit } & Paludisme & Sénégal & Pousset (2004) \\
\hline & & Fièvre & Benin & Adjanohoun (1989) \\
\hline \multirow{3}{*}{$\begin{array}{l}\text { Waltheria } \\
\text { indica }\end{array}$} & \multirow[t]{3}{*}{ Plante entière } & Stomatites & & \\
\hline & & Toux & Benin & Adjanohoun(1989) \\
\hline & & Diarrhées & Nigeria & Sofowara(1986) \\
\hline \multirow{2}{*}{$\begin{array}{l}\text { Xylopia } \\
\text { aethiopica }\end{array}$} & \multirow[t]{2}{*}{ Fruits } & hémorragies & & \\
\hline & & Toux & Sénégal & Pousset (2004) \\
\hline \multirow{3}{*}{$\begin{array}{l}\text { Zingiber } \\
\text { officinale }\end{array}$} & \multirow[t]{3}{*}{ Rhizomes } & Maux de cœurs & & \\
\hline & & Sinusite & & \\
\hline & & Toux & Inde & Ghosh (2011) \\
\hline \multirow{3}{*}{$\begin{array}{l}\text { Zizyphus } \\
\text { mauritiana }\end{array}$} & Racines & Hémorroïdes & & \\
\hline & \multirow[b]{2}{*}{ Feuilles } & Bilharziose & & \\
\hline & & Diarrhées & Burkina Faso & Zerbo (2011) \\
\hline
\end{tabular}

\subsubsection{Justification scientifique des usages des plantes dans la région de l'Azawagh}

Dans cette partie, il s'agit de la justification des usages médicinales des plantes à travers des études scientifiques (tests biologiques) déjà réalisées par d'autres auteurs ou en faisant un lien entre la composition chimique des plantes et les propriétés thérapeutiques des plantes. En effet ; chaque famille de métabolite secondaire possède une activité biologique qui serait responsable des propriétés thérapeutiques des plantes. Ce lien servirait aussi de base scientifique de l'utilisation traditionnelle des plantes sélectionnées.

\section{Acacia albida Willd. ex Delile subsp. adstringens (Mimosaceae)}

Selon les résultats du criblage chimique réalisé dans notre laboratoire (Tableau 2), les feuilles renferment des alcaloïdes, des tanins, des flavonoïdes, des saponosides, des stéroïdes et des terpènes. La tige renferme des tanins, des flavonoïdes, des saponosides, des stéroïdes et terpènes. Karoune (2016) signale également la présence dans les feuilles des alcaloïdes, tanins et flavonoïdes.

L'usage du macéré des feuilles contre les diarrhées et maux de ventre, l'usage des tiges en cas de dysenterie pourraient se justifier par les propriétés astringentes et antibactériennes des tanins (Bruneton, 1999) contenus dans les feuilles. L'usage des tiges en cas d'ictères pourrait se justifier par les propriétés antivirales des flavonoïdes signalées par Bruneton (1999). 
Acacia nilotica (L.) Willd. ex Delile var Adansoni (Mimosaceae)

Le criblage chimique effectué dans notre laboratoire (Tableau 2), a révélé la présence des tanins dans les feuilles et les gousses. Batanouny (1999) signale également que les gousses sont riches en composés phénoliques.

L'usage des gousses contre la bilharziose se justifie par les propriétés molluscides et alguicides dues aux composés phénoliques (Oumarou, 1996 ; Batanouny, 1999). L'usage des gousses contre les plaies et les aphtes se justifie par les propriétés astringentes et cicatrisantes signalées par Oumarou (1996). L'usage des gousses en cas de diarrhées et dysenterie pourrait se justifier par les propriétés antimicrobiennes rapportées par Batanouny (1999) et les propriétés astringentes signalées par Oumarou (1996).

\section{Achyrantes aspera L. (Amaranthaceae)}

Le criblage chimique effectué dans notre laboratoire (Tableau 2), a montré que les feuilles contiennent des stéroïdes et des terpènes. Les racines contiennent des tanins, des saponosides, des flavonoïdes, des quinones, des stéroïdes et des terpènes. Les fruits renferment des alcaloïdes, des saponosides, des flavonoïdes, des quinones, des stéroïdes et des terpènes. La tige contient des saponosides, stéroïdes et des terpènes. Srivastava et al. (2014) signalent que la plante entière renferme des tanins, des flavonoïdes, des stéroïdes et terpènes.

L'usage de la plante entière contre la syphilis et la fertilité féminine se justifient respectivement par les propriétés antimicrobiennes et antibactériennes dues aux tanins et les propriétés abortives et anti fertilités dues aux alcaloïdes signalées par Srivastava et al. (2014). L'usage de la plante entière contre la toux pourrait se justifier par les propriétés antiinflammatoires des flavonoïdes signalées par Bruneton (1999).

\section{Allium sativum L. (Liliaceae)}

Le criblage chimique réalisé dans notre laboratoire (Tableau 2) a révélé la présence des flavonoïdes, des saponosides, des quinones, des stéroïdes et des terpènes dans la bulbe. Londhe (2011) rapporte également que l'ail renferme des flavonoïdes, des saponosides, des huiles essentielles.

L'usage du bulbe contre les vers intestinaux dans l'Azawagh se justifie par les propriétés anthelminthiques signalées par Londhe (2011). L'usage contre les convulsions pourrait se justifier par les propriétés spasmodiques des flavonoïdes signalées par Bruneton (1999).

\section{Balanites aegyptiaca (L.) Delile (Balanitaceae)}

Le criblage chimique effectué dans notre laboratoire (Tableau 2) a montré que les feuilles renferment des saponosides, des tanins, des flavonoïdes, des stéroïdes et des terpènes. L'écorce renferme des alcaloïdes, 
des tanins, des flavonoïdes, des saponosides, des stéroïdes et des terpènes. Le fruit contient des alcaloïdes, des saponosides, des stéroïdes et des terpènes. Selon Gupta (2012) la pulpe du fruit renferme des anthocyanes, des stérols, des triterpènes, des tanins, des saponosides et l'écorce est riche en saponine, flavonoïdes, tanins, alcaloïdes et acides phénoliques.

Les usages des racines contre les morsures venimeuses et des fruits comme vermifuge se justifient respectivement par l'activité antivenimeuse et l'activité anthelminthique signalées par Tesfaye (2015). Les usages des feuilles contre les coliques et en cas de métrorragie pourraient se justifier par les propriétés astringentes et antibactériennes des tanins signalées par Bruneton (1999). L'usage des racines contre le rhume et des feuilles contre les sinusites pourrait se justifier par les propriétés antiinflammatoires des flavonoïdes signalées par Bruneton (1999).

\section{Bauhinia rufrescens Lam. (Caesalpinaceae)}

Le criblage chimique effectué dans notre laboratoire (Tableau 2) a révélé la présence dans les feuilles des tanins, des flavonoïdes, des stéroïdes et des terpènes. L'écorce renferme des tanins. Nock (2013) indiquent la présence des anthraquinones, des tanins, des flavonoïdes, des terpenoides, des stéroïdes, des phlobetanins, des résines et des alcaloïdes.

L'usage du décocté des écorces contre les diarrhées pourrait se justifier par les propriétés astringentes, l'action antibactérienne des extraits des écorces dues aux tanins et flavonoïdes signalées par Nock et al. (2013). L'usage des feuilles en cas de dysenterie se justifie par l'usage anti dysentérique des feuilles et les propriétés antimicrobiennes montrées par Nock (2013).

\section{Boscia senegalensis (Pers.) ex Poir (Capparidaceae)}

Le criblage chimique effectué dans notre laboratoire (Tableau 2) a révélé la présence des flavonoïdes, des saponosides, des stéroïdes et des terpènes dans les feuilles. Les racines renferment des alcalö̈des, des saponosides, des stéroïdes et des terpènes. Morgan (2014) rapporte que les feuilles renferment des saponosides et des terpènes.

L'usage des racines en cas de diarrhées pourrait se justifier par les propriétés astringentes et antibactériennes des tanins signalés par Bruneton (1999). L'usage des feuilles contre les maux de ventre se justifierait par les propriétés antiparasitaires signalées par Mahmout (2012) et les propriétés antibactériennes des tanins signalées par Bruneton (1999).

\section{Cadaba glandulosa Forssk. (Capparidaceae)}

Selon le criblage chimique réalisé dans notre laboratoire (Tableau 2), les feuilles renferment des alcaloïdes, des tanins, des flavonoïdes, des saponosides, des stéroïdes et des terpènes. Gohar (2010) rapporte également 
que les feuilles contiennent des tanins, des flavonoïdes, des saponines, des glucosides et des alcaloïdes.

L'usage des feuilles contre la diarrhée, la bilharziose et les maux de ventre pourrait se justifier par les propriétés astringentes et antibactériennes des tanins signalées par Bruneton (1999). De même l'usage des feuilles contre la sinusite pourrait se justifier par les propriétés antiinflammatoires des flavonoïdes signalées par Bruneton (1999).

\section{Cadaba farinosa Forssk. (Capparidaceae)}

Le criblage chimique effectué dans notre laboratoire (Tableau 2) a révélé la présence des tanins et des saponosides dans les feuilles. Umesh (2010) montre aussi la présence des composés phénoliques, des stéroïdes, des tanins, des flavonoïdes, des alcaloïdes dans les feuilles.

L'usage des feuilles en cas d'ictère pourrait se justifier par des propriétés antivirales des flavonoïdes signalées par Bruneton (1999). L'usage des feuilles contre les vers intestinaux se justifie par l'activité anthelminthique signalée par Umesh (2010).

\section{Calotropis procera (Aiton) W.T.Aiton (Asclepiadaceae)}

Le criblage chimique effectué dans notre laboratoire (Tableau 2) a révélé la présence dans les feuilles et les fleurs des tanins, des flavonoïdes, des stéroïdes et des terpènes. Hakimeh (2014) montre également que les feuilles renferment des flavonoïdes et les fleurs renferment des flavonoïdes, des anthocyanines et des stérols.

L'usage des feuilles contre la sinusite pourrait se justifier par les propriétés antiinflammatoires signalées par Nikiema (2005) et par les propriétés antiinflammatoires des flavonoïdes signalées par Bruneton (1999). L'usage des fleurs et feuilles dans le traitement de la toux et l'angine pourrait se justifier par les propriétés antitussives signalées par Nikiema (2005) et par les propriétés antiinflammatoires des flavonoïdes signalées par Bruneton (1999).

\section{Cassia italica (Mill.)Spreng (Caesalpinaceae)}

Le criblage chimique réalisé dans notre laboratoire (Tableau 2) a révélé la présence des tanins, des stéroïdes et des terpènes dans les feuilles. Les fleurs renferment des tanins, des saponosides, des quinones, des stéroïdes et des terpènes. Batanouny (1999) et Pousset (2004) ont mis en évidence des saponosides, des anthraquinones et des pigments flavonoiques dans les feuilles.

L'usage du décocté des fleurs en cas de splénomégalie se justifie par les propriétés antiinflammatoires signalées par Batanouny (1999). L'usage des 
fleurs et racines contre le ballonnement de ventre et comme laxative se justifie par les propriétés laxatives signalées par Pousset (2004).

\section{Chrozophora brocchiana (Vis.) Schweinf (Euphorbiaceae)}

Le criblage chimique effectué dans notre laboratoire (Tableau 2) a montré la présence des tanins, des flavonoïdes, des stéroïdes et des terpènes dans les feuilles. Mariod et al. (2014) ont aussi indiqué la présence des terpenoides, des flavonoïdes, des alcaloïdes, des coumarines et des tanins dans les parties aériennes.

L'usage des feuilles contre les diarrhées et la dysenterie se justifie par les propriétés antimicrobiennes signalées par Mariod et al. (2014) et les propriétés astringentes et antibactériennes des tanins signalées par Bruneton (1999).

\section{Citrillus colocynthis (L.) Schrad. (Cucurbitaceae)}

Le criblage chimique effectué dans notre laboratoire (Tableau 2) a révélé la présence des alcaloïdes, des tanins, des stéroïdes et des terpènes dans la pulpe du fruit. De même, Chawech (2015) a montré que le fruit renferme des acides phénoliques, des alcaloïdes et des flavonoïdes.

L'usage du fruit contre les dermatoses et l'usage contre les morsures de serpent pourraient se justifier respectivement par les propriétés antifongiques et les propriétés anesthésiques locales signalées par Ramanathan (2011) puis par l'activité anesthésique des alcaloïdes signalée par Bruneton (1999).

\section{Combretum glutinosum Perr. ex DC. (Combretaceae)}

Le criblage chimique réalisé dans notre laboratoire (Tableau 2) a révélé la présence des tanins, des flavonoïdes, des saponosides, des stéroïdes et des terpènes dans les feuilles. Pousset (2004) a aussi montré que les feuilles renferment des tanins galliques et des flavonoïdes.

L'usage des racines en cas de furoncle pourrait se justifier par les propriétés antiinflammatoires des flavonoïdes signalées par Bruneton (1999). L'usage des écorces en cas de dysenterie et l'usage des feuilles contre les maux de ventre pourraient se justifier par les propriétés antibactériennes et astringentes des tanins signalées par Bruneton (1999) et les propriétés antimicrobiennes signalées par Pousset (2004). L'usage des feuilles en cas d'ictère se justifie par les propriétés antivirales des flavonoïdes signalées par Bruneton (1999).

\section{Combretum micranthum G.Don (Combretaceae)}

Le criblage chimique effectué au laboratoire (Tableau 2) a révélé la présence des alcaloïdes, des tanins, des flavonoïdes, des saponosides, des stéroïdes et des terpènes dans les feuilles. Bassene et al. (1986) ont aussi 
montré que les feuilles renferment des flavonoïdes, alcaloïdes, des saponosides, des coumarines, des tanins et des triterpènes.

L'usage des feuilles en cas d'ictère, de dysenterie et contre les maux de ventre pourrait se justifier respectivement par les propriétés antivirales des flavonoïdes, les propriétés antibactériennes des tanins et les propriétés analgésiques des alcaloïdes signalées par Bruneton (1999).

\section{Commiphora Africana Engl Rich A. (Burseraceae)}

Le criblage chimique effectué dans notre laboratoire (Tableau 2) a révélé la présence des tanins, des flavonoïdes, des stéroïdes et des terpènes dans les feuilles et la tige. Adebayo et al. (2006) et Akor et Anjorin (2009) ont aussi montré que les feuilles contiennent des tanins, des alcaloïdes, des triterpènes, des stérols et des composés phénoliques.

L'usage des feuilles contre la toux se justifie par les propriétés antitussives signalées par Adebayo et al (2006) et les propriétés antiinflammatoires des flavonoïdes signalées par Bruneton (1999). L'usage des écorces contre la dysenterie pourrait se justifier par les propriétés antibactériennes signalées par Akor et Anjorin (2009), les propriétés astringentes et antibactériennes des tanins signalées par Bruneton (1999). L'usage des tiges dans le traitement des hémorragies pourrait se justifier par les propriétés astringentes des tanins signalées par Bruneton (1999).

\section{Cymbopogon schoenantus Spreng. (Poaceae)}

Le criblage chimique effectué dans notre laboratoire (Tableau 2) a montré la présence des tanins, des flavonoïdes, des saponosides, des stéroïdes et des terpènes dans les parties aériennes. Samate (1995) ont aussi montré que la plante renferme des huiles essentielles, des tanins, des flavonoïdes.

L'usage des feuilles en cas de myalgie, odontalgie et courbatures se justifie par les propriétés sédatives signalées par Samate (1995). L'usage en cas d'anurie se justifie par les propriétés diurétiques signalées par Kokwaro (1976).

\section{Eugenia caryophyllata Thunb. (Myrthaceae)}

Le criblage chimique réalisé dans notre laboratoire (Tableau 2) a révélé la présence des tanins, des flavonoïdes, des saponosides, des stéroïdes et des terpènes dans les feuilles. Roman (2015) a de même indiqué que les feuilles renferment des flavonoïdes, de l'eugénol et des tanins.

L'usage des feuilles en cas de sinusites et en cas de rhume pourrait se justifier par les propriétés antiinflammatoires dues à l'eugénol (Roman, 2015). L'usage des feuilles en cas des hémorragies pourrait se justifier par les propriétés astringentes des tanins signalées par Bruneton (1999). 


\section{Euphorbia hirta L. (Euphorbiaceae)}

Le criblage chimique réalisé dans notre laboratoire (Tableau 2) a révélé la présence des alcaloïdes, des tanins, des flavonoïdes, des saponosides, des stéroïdes et des terpènes dans la plante entière. Pousset (2004) révèle également la présence des flavonoïdes, des alcaloïdes, des tanins et des polyphénols.

L'usage de la plante en cas d'asthme, bronchite et maladies respiratoires se justifie par les propriétés antiasthmatiques et antiinflammatoires signalées par Pousset (2004). L'usage des feuilles en cas de blennorragies pourrait se justifier par les propriétés antiinfectieuses des tanins signalées par Bruneton (1999).

\section{Guiera senegalensis J.F. Gmel (Combretaceae)}

Le criblage chimique réalisé dans notre laboratoire (Tableau 2) a révélé la présence des alcaloïdes, des tanins, des flavonoïdes et des saponosides dans les feuilles. Usman (2015) signale aussi que les feuilles renferment des alcaloïdes, tanins et flavonoïdes.

L'usage des feuilles contre la fièvre se justifie par les propriétés antipyrétiques et fébrifuges signalées par Kerharo et al. (1974). L'usage des feuilles contre l'ictère se justifie par les propriétés antivirales des flavonoïdes signalées par Bruneton (1999). L'usage des feuilles contre la dysenterie pourrait se justifier par les propriétés antimicrobiennes signalées par Usman et al. (2015) et par les propriétés antibactériennes et astringentes des tanins signalées par Bruneton (1999).

\section{Lawsonia inermis L. Lythraceae)}

Le criblage chimique réalisé au laboratoire (Tableau 2) a révélé la présence des alcaloïdes, des tanins, des flavonoïdes, des saponosides, des quinones, des stéroïdes et des terpènes dans les feuilles. Semwal (2014) signale aussi la présence des flavonoïdes, des tanins, des naphtoquinones et des alcaloïdes.

L'usage des feuilles dans le traitement des plaies se justifie par l'action curative des plaies due à la lawsone signalée par Semwal (2014). L'usage des feuilles dans le traitement des furoncles se justifie par l'activité anti tumorale des flavonoïdes signalée par Bruneton (1999).

\section{Maerua crassifolia Forssk. (Capparidaceae)}

Le criblage chimique réalisé dans notre laboratoire (Tableau 2) a révélé la présence des alcaloïdes, des tanins, des flavonoïdes, des saponosides, des stéroïdes et des terpènes dans les parties aériennes. Akuodor et al. (2014) signalent aussi que la plante entière renferme des alcaloïdes, des saponines, des tanins, des terpenoides, des flavonoïdes et des stéroïdes. 
L'usage des feuilles en cas d'ictères pourrait se justifier par les propriétés antivirales des flavonoïdes signalées par Bruneton (1999). L'usage des feuilles en cas de paludisme, en cas de diarrhées et contre la toux se justifient respectivement par les propriétés antipyrétiques et anti malariques, les propriétés anti diarrhéiques et les propriétés antiinflammatoires des feuilles rapportées par Akuodor et al. (2014).

\section{Ocinum canum Sims (Solanaceae)}

Le criblage chimique réalisé dans notre laboratoire (Tableau 2) a révélé la présence des alcaloïdes, des tanins, des flavonoïdes, des saponosides, des stéroïdes et terpènes dans les feuilles. Kosni et al. (2015) rapportent de même que les feuilles renferment des saponines, des flavonoïdes, des alcaloïdes, des tanins, et une huile essentielle dont le composé majoritaire est le 1,8-cineole (Djibo, 2004).

L'usage de la poudre des feuilles contre le rhume et l'usage de la plante entière contre la toux pourraient se justifier par l'activité antiinflammatoire des flavonoïdes signalée par Bruneton (1999).

\section{Sclerocarya birrea (A.Rich.) Hochst. (Labiaceae)}

Le criblage chimique réalisé dans notre laboratoire (Tableau 2) a révélé la présence des tanins, des flavonoïdes, des saponosides, des quinones, des stéroïdes et des terpènes dans les feuilles. Les écorces renferment des tanins, des flavonoïdes, des saponosides, des quinones, des stéroïdes et terpènes.

L'usage des feuilles en cas de diarrhées, l'usage des écorces en cas de dysenterie et maux de ventre se justifient par les propriétés anti diarrhéiques, anti bactérienne et antiamibiennes signalées par Mariod (2012). L'usage des écorces en cas d'ictère pourrait se justifier par les propriétés antivirales des flavonoïdes signalées par Bruneton (1999).

\section{Tamarindus indica L. (Asclepiadaceae)}

Le criblage chimique réalisé dans notre laboratoire (Tableau 2) a montré la présence des tanins et des flavonoïdes dans les feuilles. Les écorces contiennent des alcaloïdes, des tanins, des flavonoïdes et des saponosides. Le fruit contient des flavonoïdes, des saponosides, des stéroïdes et des terpènes. Guissou (2010) rapporte aussi que les écorces contiennent des flavonoïdes, des saponosides, des tanins, des alcaloïdes; les feuilles contiennent des flavonoïdes et le fruit contient des monoterpènes.

L'usage des fruits en cas de fièvres et l'usage des racines en cas de métrorragie pourraient se justifier respectivement par les propriétés antivirales des flavonoïdes et astringentes des tanins signalées par Bruneton (1999). L'usage des fruits en cas de paludisme se justifie par les propriétés antiplasmodiques liées aux terpenoides selon Guissou (2010). 


\section{Tribulus terrestris L. (Caesalpinaceae)}

Le criblage chimique réalisé dans notre laboratoire (Tableau 2) a révélé la présence des alcaloïdes, des flavonoïdes, des saponosides, des stéroïdes et des terpènes dans les feuilles. Samy (2013) rapporte également la présence des alcaloïdes, des flavonoïdes, des stéroïdes et terpènes dans les feuilles.

L'usage des feuilles en cas d'hémorragie se justifie par les propriétés astringentes signalées par Boulos (1983) et par Samy (2013). L'usage en cas d'impuissance sexuelle se justifie par l'usage contre l'impotence, l'infertilité et les propriétés aphrodisiaques signalées Samy (2013) et l'usage comme aphrodisiaque et pour croitre la spermatogenèse rapportée par Boulos (1983).

\section{Waltheria indica L. (Malvaceae)}

Le criblage chimique réalisé dans notre laboratoire (Tableau 2) a révélé la présence des alcaloïdes, des flavonoïdes, des saponosides, des quinones, des stéroïdes et des terpènes dans la plante entière. Zongo (2013) signalent aussi que la plante entière renferme des alcaloïdes, des flavonoïdes, des tanins, des stérols, des terpènes, des saponines et des anthraquinones.

L'usage de la plante entière contre la toux et les stomatites se justifient respectivement par les propriétés antiinflammatoires dues à la quercetine, tiliroside et epicatechine signalées par Zongo (2013).

\section{Xylopia aethiopica (Dunal) A.Rich (Sterculiaceae)}

Le criblage chimique réalisé dans notre laboratoire (Tableau 2) a révélé la présence des alcaloïdes, des flavonoïdes, des saponosides, des stéroïdes et des terpènes dans les fruits. Ezekwesili (2010) signale aussi que le fruit renferme des alcaloïdes, des saponosides, des tanins, des anthraquinones, des stéroïdes. De même, Aguoru et al. (2016) rapportent que le fruit renferme des flavonoïdes et des huiles essentielles.

L'usage des fruits contre la toux se justifie par les propriétés sédatives contre la toux signalées par Ezekwesili (2010) et les propriétés antiinflammatoires des flavonoïdes signalées par Bruneton (1999).

\section{Zingiber officinale Roscoe (Zingiberaceae)}

Le criblage chimique réalisé dans notre laboratoire (Tableau 2) a révélé la présence des flavonoïdes, des stéroïdes et des terpènes dans le rhizome. Les usages du rhizome contre les maux de cœurs, les sinusites et la toux se justifient respectivement par les propriétés cardioprotectrices, antiinflammatoires dues au gingérol (Ghosh, 2011).

\section{Zizyphus mauritiana Lam. (Annonaceae)}

Le criblage chimique réalisé dans notre laboratoire (Tableau 2) a révélé la présence des tanins, des flavonoïdes, des saponosides, des stéroïdes et des 
terpènes dans les feuilles. La tige renferme des alcaloïdes, des tanins, des flavonoïdes, des stéroïdes et terpènes. Le fruit renferme des alcaloïdes, des tanins, des flavonoïdes, des stéroïdes et des terpènes. Hussain et al. (2008) ont en plus signalé que l'écorce renferme des alcaloïdes, des triterpènes, des saponosides, des tanins, des flavonoïdes.

L'usage des feuilles en cas de diarrhées et bilharziose se justifie par l'activité anti diarrhéique signalée par Gueda (2005) et les propriétés astringentes et antibactériennes des tanins rapportées par Bruneton (1999).

\section{Conclusion}

Une bibliographie effectuée sur l'usage médicinale de chacune de ces trente (30) plantes a permis de constater que plusieurs pays utilisent ces mêmes plantes dans le traitement des mêmes pathologies que dans la zone de l'Azawagh. Il s'agit notamment de Benin, Burkina Faso, Sénégal, Inde, Mauritanie, Mali, Egypte, Soudan, Nigeria, Djibouti, Madagascar et Tunisie. De plus, des résultats scientifiques tirés des travaux de plusieurs auteurs ainsi que le criblage chimique réalisé dans notre laboratoire sont en faveur de l'existence des substances bioactives contre les différentes pathologies où les trente (30) plantes sont utilisées. Les similarités d'usages et l'existence des composés chimiques responsables des effets thérapeutiques observés permettent de fournir une base scientifique de l'utilisation thérapeutique traditionnelle des plantes étudiées. Cette base scientifique apporte une justification sur l'utilisation de ces trente (30) plantes médicinales par les tradipraticiens de la zone de l'Azawagh. De telles investigations seront conduites sur les autres espèces végétales du Niger. Des études in vitro et/ou in vivo sont envisagées pour l'évaluation de certaines propriétés biologiques. De même, des investigations phytochimiques en vue d'isoler les molécules responsables des activités ciblées seront menées.

\section{Remerciements}

Nous remercions les différents centres de documentation à savoir le ministère de l'agriculture et l'élevage, les bibliothèques de la Faculté des Sciences et Techniques, la Faculté d'Agronomie, l'IRSH, l'AGRYMET. Nous remercions également le CNRS (Gif-Sur-Yvette) pour son appui financier.

\section{References:}

1. Hussain, M. N. Khan, Z. Iqbal, E U. Ghafoor and I. Ahmad (2011). In vitro screening of Ziziphus mauritiana and Terminalia arjuna for their anthelmintic activity. The journal of animal and plant science 21(4) : 781-786. 
2. ABDALLAH AH (2013). Medicinal plants and their uses by the people of Randa, Djibouti. Journal of ethnopharmacology 148:701713

3. ADEBAYO AA, R.Aliyu et I.G Garba (2006). The effects of ethanolic leaf extract of Commiphora africana(Burseraceae) on lipid profil in rats. International journal of pharmacology 2(6) : 618-622.

4. ADJANOHOUN E.J (1985). Médecine traditionnelle et pharmacopée, contribution aux études ethnobotaniques et floristiques au MALI, ACCT.

5. ADJANOHOUN E.J (1989).Médecine traditionnelle et pharmacopée, contribution aux études floristiques au BENIN. ACCT.

6. ADJANOHOUN E.J (1999). Programme des ressources génétiques forestières en Afrique au sud du Sahara (programme SAFORGEN). Compte rendu de la première réunion du réseau. Disponible sur www.futureharvest.org.

7. B P Fabregues (1972). Lexiques des noms vernaculaires des plantes du Niger. Institut National de la Recherche Agronomique du NigerNiamey.

8. BARBELET S (2015). Le giroflier : historique, description, utilisation de la plante et son huile essentielle. Thèse de doctorat en pharmacie. Université de Lorraine (France).

9. BASSENE E (1986). Plantes medicinales africaines. Les alcaloides de Combretum micranthum. Ann pharmaceutiquc françaises 44 :191-196

10. BATANOUNY KH (1999). A guide to medicinal plants in North Africa.

11. BERNUS E (1970). Récits historiques de l'Azawagh. Bulletin de 1'I.F.A.N. ser.B n²

12. BOITEAU P (1987). Médecine traditionnelle et pharmacopée. Précis de matière médicale malgache. ACCT.

13. BOULOS L (1983). Medicinal plants of North Africa.

14. BRUNETON J (1996). Pharmacognosie Phytochimie, plantes médicinales.

15. U. Aguoru, C. Pilla and J. O. Olasan (2016). Phytochemical criblage of Xylopia aethiopica with emphasis on its medicinally active principles. Academic journal 10 (22) : 306-309.

16. Cissé A, Guèye M, Koma S, Akpo LE, (2016). Ethnobotanique des plantes médicinales chez les bergers peuls de Widou Thiengoly de la commune de Téssékéré (Ferlo-Nord Sénégal).

17. Daniel Kosni, Elias Nchiwan Nukenine, Katamssadan Haman Tofel (2015). Efficacy of Cameroonian Ocimum canum Sims (Lamiaceae) leaf extract fractions against Callosobruchus maculatus (F.) 
(Coleoptera: Chrysomelidae), infesting Bambara groundnut. Journal of Entomology and Zoology Studies 3(5):487-494.

18. Dibong, S, D., Mpondo, E., Ngoye A., Kwin, M, F., (2011). Etude de l'efficacité et de la sélectivité d'Imazétbapyr 240 g 11 contre les adventices du niébé (Vigna unguiculata (L.)) et ses effets sur les propriétés chimiques du sol.

19. DJIBO Alpha Keita (2004). Composition chimique de l'huile essentielle d'Ocinum americanum Linn. Syn. O.canum Sims. C.R. Chimie 7 :1033-1037.

20. EZEKWESILI CN (2010). Investigation of the chemical composition and biological activities of Xylopia aethiopica(Annonaceae). African Journal of biotechnology 9 (43) :7352-7356.

21. FAD (Fonds Africains pour le Développement) (2001). Projet d'amélioration de la qualité des soins de santé. Rapport d'évaluation. Niger.

22. G C Akuodor, KC Ckilaka, JL Akpan, ED Ogiji (2016). Antibacterial and antioxydant activities of methanolic leaf extract of Maerua crassifolia. Journal of applied pharmaceutical service 5(10).

23. G. C. Akuodor, J. A. Ibrahim, J. L. Akpan, A. U. Okorie (2014). Phytochemical and anti-diarrhoeal properties of methanolic leaf extract of Maerua crassifolia. European journal of medicinal plants 4(10) : 1223-1231.

24. GHOSH AK (2011). Zingiber officinale : a natural gold. Journal of pharma and biosciences 2(1) :283-294.

25. Giani (2006). Médecines traditionnelles et développement local : ethodologies et approches. Universitas Forum 2(2) 1-21.

26. GNOULA C (2009). Etude des propriétés cytotoxiques et anti radicalaires des extraits des feuilles et de galles de Guiera senegalensis. Science et technique $32: 9-23$

27. GOHAR AA (2010). Flavonol glycosides from Cadaba gandulosa. Z.naturforsh $57: 216-220$.

28. GUEDA BA SH (2005). Etude de la Phytochimie et des activités biologiques de Zizyphus mauritiana Lam (Rhamnaceae) utilisée dans le traitement du diabète et de l'hypertension artérielle en Mauritanie. Thèse de doctorat en pharmacie. Université de Bamako(Mali).

29. GUISSOU I (2010). Etudes des propriétés pharmacologiques du fruit de Tamarindus indica utilisé comme laxatif et antipaludique en médecine traditionnelle. Fruit, vegetable, and cereal science and biotechnology $4: 31-35$

30. GUPTA S.C (2012). Pharmacognostical and phytochemical evaluation of Balanites aegyptiaca Linn Delile stem bark. Internatinal research Journal of pharmacy 3(7):169-174. 
31. HAKIMEH Olumi (2014). Phytochemistry and ethno-pharmaceuticals of Calotropis procera. Ethno-pharmaceuticals products 1(2):1-8.

32. HASAN S (2014).Pharmacological and medicinal uses of Achyranthes aspera. International Journal of sciences, environnement and technology 3(1): :123-129

33. HASSAN HA (2012).Chemical composition and antimicrobial activity of the crude extracts isolated from Zingiber officinale by differents solvants. Pharmaceut anal acta $3: 1-5$.

34. HMEIADA Ould Mohamed vall (2009). Contribution à l'étude des plantes médicinales de Mauritanie. Ann univ Lome (Togo) p9-27.

35. HUSSAIN (2011). In vitro criblage of Ziziphus mauritiana and Terminalia arjuna for their anthelmintic activity. The journal of animal and plant science 21(4) : 781-786

36. IKHIRI Khalid, ILAGOUMA Amadou et GUERO Djimrao (2000). Etudes sur la préparation d'un programme de promotion de la pharmacopée traditionnelle de la zone d'intervention du PROZOPAS, KRB ingénieurs rapport définitif. Université A.Moumoni (Niger).

37. J.S. AKOR AND T.S. ANJORIN (2009). Phytochemistry and antimicrobial studies of Commiphora africana. International journal of agriculture and biology 11(6) : 795-797.

38. KAROUNE S (2016). Etude Eco physiologique et phytochimique de deux espèces d'Acacia : Acacia albida et Acacia raddiana. Thèse de doctorat en science. Université des Frères Mentouri Constantine(Algérie).

39. KERHARO J et J.G ADAM (1974). Pharmacopée sénégalaise traditionnelle; plantes médicinales et toxiques. Ed Vigot, PARIS.

40. Koffi N'GUESSAN, Beugré KADJA, Guédé N. ZIRIHI, Dossahoua TRAORÉ, Laurent AKÉ-ASSI (2009). Screening phytochimique de quelques plantes médicinales ivoiriennes utilisées en pays Krobou (Agboville, Côte-d'Ivoire). Sciences \& Nature Vol. $6 \mathrm{~N}^{\circ} 1: 1-15$.

41. KOKWARO JO (1976). Medicinal plants of East Africa. East african litterature bureau

42. Lee K.H., (2004). Current developments in the discovery and design of new drug candidates from plant natural product lead, Journal of Natural Products 67: 273-283p.

43. LONDHE VP (2011). Role of Garlic in various diseases : An review. Journal of pharmaceutical research and opinion 1(4) :129-134.

44. MAHMOUT Yaya (2012). In vitro anti-hyperglycaemic effect of glucocapparin isolated from the seeds of Boscia senegalensis (Pers) Lam Ex Poiret. African journal of biotechnology 11(23) :6345-6349. 
45. MARIOD AA (2012). Sclerocarya birrea : biochemichal, composition, nutritional and medicinal uses. A review. Natural products : research view vol 1. P29-118. www.sustech.edu.

46. MARIOD AA (2014). Review: biochemical composition and medicinal uses of chrozophora genus. International journal of pharmacy. Review and research 4 (41):227-232.

47. MORGAN AM (2014). A New Flavonol Glycoside from the Leaves of Boscia senegalensis.

48. Nacoulma-Ouédraogo (1996), Plantes médicinales et pratiques médicales traditionnelles au Burkina Faso: cas du Plateau central, Thèse de Doctorat ès Sciences Naturelles, Université de Ouagadougou, (Burkina-Faso), 605 pp.

49. NIKIEMA WP (2005). Propriétés pharmaco chimique de Calotropis procera (Asclepidiaceae) récolté au Mali : étude préclinique des effets anti inflammatoires et anti microbiennes des extraits des écorces des racines. Thèse de doctorat. Faculté de pharmacie, médecine, et d'odontostomatologie. Université de Bamako(Mali)

50. NOCK IH (2013). Hypoglycaemic Activity and Nephro-Protectective Effect of Bauhinia rufescens in Alloxan-Induced Diabetic Rats. International journal of advances in pharmacy biology and chemistry Vol. 2(1).

51. OUMAROU A (1996). Contribution à l'étude de l'activité spasmolytique des fruits murs et des écorces du tronc d'Acacia nilotica var Adansonii (mimosacée). Thèse de doctorat : vétérinaire. Université Anta Diop de Dakar-école inter-Etats des sciences et médecine vétérinaire (EISMV)

52. POUSSET J L (2004). Plantes médicinales d'Afrique. Comment les reconnaitre et les utiliser? EDISUD

53. POUSSET JL (1989). Plantes médicinales africaines. Utilisation pratique. Tome 1 et 2. Ellipses. ACCT

54. Rachid Chawech, Raoudha Jarraya, Cynthia Girardi, Marieke Vansteelandt and Nicolas Fabre (2015). Cucurbitacins from the leaves of Citrulus colocynthis L Schrad. Molecules $20: 18001-18015$

55. RAMANATHAN T (2011). Antimicrobial effect of coastal medicinal plant. Citrulus colocynthis against pathogenic microorganismes. African Journal of pure and applied chemistry vol 5(5) p119-122.

56. ROMAN L (2015). Eugenia caryophyllata Thunberg - A MIRACULOUS HERB.

57. SAMATE AD (1995). Composition chimique des huiles essentielles de trois espèces du genre Cymbopogon (poaceae), Cymbopogon citratus, Cymbopogon giganteus, Cymbopogon schoenantus du Burkina Faso. Journal de la SOACHIM P.43. 
58. SAMY MN (2013). Pharmacognostical studies on flower of Tribulus terrestris. Journal of pharmacognosy and phytochemistry.

59. SEMWAL RB (2014). Lawsonia inermis L. (henna): Ethnobotanical, phytochemical and pharmacological aspects. Journal of Ethnopharmacology vol 155 p80-103.

60. SERENE A (2008). Propriétés thérapeutiques des plantes à tanins du Burkina Faso. Pharmacopée et médecine traditionnelle africaines vol $15 \mathrm{p} 41-49$

61. SOFOWARA A (1986). The state of medicinal plants research in NIGERIA

62. SRIVASTAVA (2014). Achyrantes aspera. An important medicinal plant : a review. J. Nat plant ressour.Vol1(1) p1-14.

63. TESFAYE A (2015). Balanites aegyptiaca Del.multipurpose tree a prospectrive review. International journal of modern chemistry and applied science 2(3) p189-194.

64. UMESH BT (2010). Pharmacognostic and phytochemical investigation on roots of Cadaba farinosa Forsk. International journal of pharma and bioscience vol 1(2) p1-13.

65. USMAN AA (2015). Antimicrobial and phytochemical of the bioactive fractions of Guiera senegalensis. Journal of pharmacognosy and phytochemistry vol 3(6) p 106-111.

66. Yves-Alain BÉKRO, Janat A. MAMYRBEKOVA BÉKRO, Boua B. BOUA, Fézan H. TRA BI \& Ehouan E. ÉHILÉ (2007). Étude ethnobotanique et criblage phytochimique de Caesalpinia benthamiana (Baill.) Herend. et Zarucchi (Caesalpiniaceae). Sciences \& Nature Vol. $4 \mathrm{~N}^{\circ} 2: 217-225$.

67. ZERBO P (2011). Plantes médicinales et pratiques médicales au Burkina Faso : cas de Sanan. Bois et forêts des tropiques $N^{\circ} 301$ P41

68. ZONGO F (2013). Botany, traditional uses, phytochemistry and pharmacology of Waltheria indica. A review. Journal of ethnopharmacology vol 148 p14-26. 